\title{
LA INTRODUCCIÓN DE PARODIA COMO GÉNERO HUMORÍSTICO EN EL AULA DE E/L2 ${ }^{1}$
}

\section{INTRODUCTION TO PARODY AS A HUMOROUS GENRE IN THE SPANISH AS A SECOND LANGUAGE CLASSROOM}

\author{
Esther Linares Bernabéu \\ Universidad de Alicante, España \\ esther.linares@ua.es
}

\begin{abstract}
Resumen:
El presente trabajo de investigación nace de la voluntad de examinar las posibilidades de uso de géneros humorísticos como la parodia en el aula de español segunda lengua. Considerando el humor verbal como un instrumento de mejora en el desarrollo de la competencia lingüística y sociocultural de los estudiantes de español como L2/LE, se llevará a cabo un análisis cuantitativo y cualitativo de los datos obtenidos con 71 estudiantes extranjeros que residen en España, a fin de comprobar la viabilidad de uso de este tipo de texto humorístico. Además, examinaremos qué factores influyen en el reconocimiento, comprensión y apreciación del discurso humorístico, poniendo nuestro foco de atención en variables como el grado de inmersión lingüística, el tiempo de estancia en España, el nivel de lengua, y el género y la edad del alumno.
\end{abstract}

Palabras clave: humor verbal, E/L2, parodia, competencia humorística, inmersión lingüística.

\begin{abstract}
:
This research originates from the purpose of studying the possibilities of using humorous genres, such as parody, in the Spanish as a Second Language classroom. Bearing in mind that humour is an enhancing tool for developing linguistic and sociocultural competences in non-native Spanish learners, a quantitative and qualitative analysis of the data collected in 71 learners will lead us to ascertain the viability of using this type of genre. Furthermore, we aim to shed light on factors that affect the recognition, understanding and appreciation processes, with a focus on variables such as the degree of language immersion, the length of residence in Spain, the language level, and the learner's gender and age.
\end{abstract}

Keywords: verbal humour, Spanish as L2, parody, humour competence, linguistic immersion.

Recibido: 03 de septiembre de 2018

Aceptado: 30 de septiembre de 2018

\footnotetext{
${ }^{1}$ La investigación recogida en el presente artículo ha sido posible gracias al contrato predoctoral financiado por la Conselleria d'Educació, Investigació, Cultura i Esport de la Generalitat Valenciana a través del Proyecto de investigación Humor de género: Observatorio de la identidad de mujeres y hombres a través del humor (PROMETEO/2016/052 IP Leonor Ruiz Gurillo).
} 


\section{Introducción}

A nivel pedagógico, el sentido del humor ha sido reconocido como un apoyo en el aula (Bell y Pomerantz 45). De hecho, el proceso de aprendizaje de una segunda lengua implica en ocasiones cierta ansiedad y temor ante lo desconocido, lo cual se puede reducir drásticamente introduciendo algunas dosis de humor; ya que el humor promueve un ambiente de aprendizaje distendido y favorece la cohesión del grupo (Jefferson 369, Vivanco 338, Schmitz 111). Además, el humor es una herramienta ideal para conseguir involucrar al alumnado en las actividades y aumentar su motivación y participación en tareas que en un principio resultarían tediosas (Bell 241).

Sin embargo, pese a las positivas implicaciones pedagógicas del humor, poco se sabe, hasta la fecha, sobre qué géneros humorísticos son más convenientes para usar en el aula de español como segunda lengua (en adelante, E/L2), contexto en el que los estudiantes se benefician de cierto grado de inmersión linguiística ${ }^{2}$. Así pues, el propósito de este estudio es demostrar los beneficios que aporta la inclusión de humor verbal al aula de ELE, poniendo el foco de atención en la introducción de la parodia, como género humorístico, dentro de los contenidos. Para ello, examinaremos los diversos factores que podrían influir a la hora de alcanzar la madurez en la competencia humorística cuando se trata de usuarios no nativos. Asimismo, nos proponemos también estudiar cómo inciden las variables en la comprensión del material humorístico audiovisual y en la apreciación del mismo. Por último, a través del prisma de la apreciación del humor, analizaremos cuál es la actitud del oyente extranjero ante el humor verbal en español ${ }^{3}$ y cómo es su reacción frente a géneros humorísticos como la parodia.

\footnotetext{
${ }^{2}$ De acuerdo con el Diccionario de términos clave de ELE, se entiende por inmersión lingüística el programa de enseñanza de una segunda lengua en el que alguna de las materias del currículo escolar (o todas ellas) se estudia en una lengua que no es la L1 de los estudiantes. El objetivo último de un programa de inmersión es que los aprendientes sean competentes en ambas lenguas, es decir, sean bilingües. De este modo se crea en el aula un contexto de adquisición en el que la L2 se usa comunicativamente en actividades académicas, practicando las diferentes destrezas lingüísticas en textos y temas propios de la asignatura en cuestión. Para más información, puede consultarse el siguiente enlace:

https://cvc.cervantes.es/ensenanza/biblioteca_ele/diccio_ele/diccionario/inmersionlinguistica.htm

${ }^{3}$ Cabe señalar que las muestras humorísticas presentadas eran ejemplos de español peninsular.
} 
A su vez, los objetivos de este trabajo se apoyan en dos principales hipótesis. En primer lugar, se parte de que las marcas e indicadores ${ }^{4}$ lingüísticos y extralingüísticos que acompañan al discurso humorístico son las pistas metapragmáticas que ayudan a los oyentes/lectores no nativos a inferir el humor y resolver la incongruencia. Con tal de comprobar esta hipótesis y contradecir así las opiniones acerca de la irresoluble problemática que supone entender el humor en otro idioma (Bell 247, Attardo y Bell 423), en esta investigación se expondrán datos reales extraídos de 71 adolescentes extranjeros a través de diversas fuentes analíticas ( $(3)$. En esta línea, una segunda hipótesis general sería que variables como la lengua materna de los estudiantes, el género, el nivel en la L2, el tiempo que lleve el aprendiz en nuestro país, el grado de inmersión lingüística, o el curso académico condicionan e inciden directamente en los procesos de reconocimiento, entendimiento y apreciación del humor verbal.

Para alcanzar nuestros objetivos y confirmar dichas hipótesis, este trabajo somete a análisis un corpus de respuestas sobre la percepción, comprensión y apreciación del humor en E/L2, en relación con cuatro géneros humorísticos diferentes. En concreto, el estudio ha contado con 71 informantes extranjeros, de edades comprendidas entre los 12 y los 18 años, que se encuentran en nuestro país realizando sus estudios de Educación Secundaria Obligatoria (en adelante, ESO) o Bachillerato.

\section{Marco Teórico}

\subsection{El humor verbal}

El humor es un fenómeno pragmático que ha sido estudiado desde tiempos inmemoriales por parte de disciplinas tan dispares como la antropología, la psicología, la educación, la medicina o la economía. Sin embargo, el ámbito de estudio desde el que parte la presente investigación es el de la lingüística.

Desde esta perspectiva, tendremos como referente la Teoría General del Humor Verbal, propuesta por Attardo y Raskin (1991) y revisada posteriormente por Attardo (1994, 2001) y Ruiz Gurillo (2012), ya que es un modelo eficaz para proporcionar una explicación lingüística del humor. Dicha teoría se asienta en seis premisas que se conocen

\footnotetext{
${ }^{4}$ Obsérvese una tipología de estos en Ruiz Gurillo (2012); Ruiz Gurillo y Alvarado Ortega (eds.), (2013).
} 
como recursos de conocimiento (knowledge resources) y que actúan de forma jerárquica. De acuerdo con la TGHV, la presencia de estos seis recursos, en cualquier texto escrito u oral, nos facilita la identificación de estos como humorísticos. Sucintamente, los seis requisitos son:

- Oposición de guiones: "a large chunk of semantic information surrounding the word or evoked by it” (Raskin 81). Esta condición es un resquicio de la Teoría Semántica del Humor basada en guiones (en adelante, TSHG) elaborada por Victor Raskin en 1985. Raskin y Attardo defienden que para reconocer un texto como humorístico, debe darse en primer lugar una oposición entre dos esquemas o guiones. De acuerdo con la TGHV, este contraste genera una incongruencia que al resolverse desencadena normalmente en un efecto hilarante. Si bien no debemos entender la oposición como una antítesis, sino como un reemplazamiento del guión serio por el guión humorístico. Como ha defendido la LC, esta contraposición de guiones se observa fácilmente en los chistes enlatados, pero es más compleja apreciarla en el humor espontáneo o en otros géneros humorísticos más extensos.

- Mecanismos lógicos: son los vehículos durante el proceso inferencial que contribuyen a la resolución de la incongruencia generada por el contraste entre los dos guiones. Dichos mecanismos, de acuerdo con la taxonomía de Attardo, Hempelmann y Di Maio (2002), pueden ser sintagmáticos, como la yuxtaposición, el quiasmo y el paralelismo, o basados en el razonamiento, como el razonamiento correcto basado en una premisa incorrecta, la falsa analogía y el metahumor.

- Situación: supuesto por el cual el contexto es un elemento imprescindible para comprender el humor. El lugar en el que se produzca el humor, así como los participantes envueltos en el mismo, determinarán que se consiga el efecto humorístico buscado, o de lo contrario sea humor fallido.

- Meta o blanco de burla: El objeto de burla no es un requisito esencial, ya que podemos encontrarnos con textos humorísticos que carecen de un blanco al que vaya dirigido la broma. No obstante, en el caso de existir, suele normalmente aludir a estereotipos individuales o de grupos sociales. 
- Estrategias narrativas: Hacen referencia a los géneros que emplea el texto como táctica para ocasionar humor, entre ellos podemos distinguir los géneros prototípicamente humorísticos y los géneros serios que pueden encerrar ciertos rasgos cómicos. Ello influirá en el grado de espontaneidad y planificación del texto y, por ende, en el registro. Así pues, ateniendo a la revisión de Ruiz Gurillo (2012), se deberá prestar atención a rasgos como la inmediatez y dinamismo dependiendo de si es un texto oral o escrito. Del mismo modo, Ruiz Gurillo (2012: 38-39) defiende que conviene conocer la tipología textual, es decir, saber si se trata de un texto narrativo, expositivo-argumentativo, o conversacional, puesto que ello reflejará la actitud del hablante/escritor e influirá también en el registro empleado.

- Lenguaje: el usuario utiliza un determinado lenguaje, es decir, unos particulares elementos fónicos, léxicos y morfosintácticos que sustentan el texto humorístico. Si atendemos a la nomenclatura desarrollada por el grupo de investigación GRIALE ${ }^{5}$, estas elecciones lingüísticas (léxicas, morfosintácticas y fónicas) serían indicadores de humor, entendidos estos como procedimientos de por sí humorísticos (Ruiz Gurillo 2010, 2012, 2014). Asimismo, en la revisión de la TGHV, Ruiz Gurillo (2012) alude a elementos paralingüísticos, kinésicos y tipográficos como marcas que acompañan al humor y colaboran en su inferencia.

Si atendemos a lo defendido en nuestra primera hipótesis, el lenguaje será el recurso de conocimiento clave que facilite la inferencia del humor. La reflexión consciente de los estudiantes sobre el uso y función de las marcas e indicadores del discurso humorístico contribuirá al desarrollo de su competencia metapragmática y humorística en E/L2. Será interesante ver cómo estos elementos lingüísticos y extralingüísticos sirven de pista al oyente/lector para identificar la incongruencia generada por la oposición de guiones e identificar el mecanismo lógico empleado por el hablante/escritor para resolverla. Además, a ello colaborarán también la situación, el blanco de burla y las estrategias narrativas empleadas en las muestras que presentamos en este estudio.

\footnotetext{
${ }^{5}$ Grupo de Investigación sobre la ironía y el humor en español del Departamento de Filología Española, Lingüística General y Teoría de la Literatura de la Universidad de Alicante. Para más datos sobre el grupo de investigación GRIALE, puede consultarse su web: http://dfelg.ua.es/griale/.
} 


\subsection{El uso del humor en el aula de e/12}

La enseñanza de español como L2/LE no ha prestado la atención necesaria al desarrollo de la competencia humorística. Sin embargo, creemos que el trabajo de esta competencia repercutiría directamente en una mejora de la competencia comunicativa y de las habilidades sociales de los aprendices. Si dotamos a nuestros estudiantes de las herramientas para comprender cómo funciona el humor en español peninsular, estaremos no solo contribuyendo al aprendizaje de español, sino también mejorando su capacidad de apreciación del humor. A pesar de la carencia de estudios empíricos en relación con el humor verbal en el aula de segundas lenguas, es de recibo resaltar a Nancy Bell, ya que, dentro del estudio del humor en hablantes no nativos, constituye una figura precursora, concretamente en estudiantes de inglés como LE. Entre sus aportaciones a este campo, destaca el estudio sobre las causas de humor fallido en el alumnado extranjero (Bell y Attardo 440; Vivanco 338, Bell 176). En particular, Attardo y Bell (2010) crearon una tipología en la que se recogen diversas razones por las que fracasa el humor en hablantes de inglés como LE. En ella observamos cómo uno de los posibles motivos es la incapacidad del oyente para procesar el lenguaje figurado y entender determinadas palabras. De este modo, si el alumnado no cuenta con un amplio repertorio léxico-semántico y una adecuada competencia pragmática, resultará imposible la resolución de la incongruencia y, por ende, la apreciación del humor.

Sin duda, el reconocimiento y comprensión del humor en una L2 son ejercicios cognitivos complejos que requieren de una preparación previa en el aula. Por ello, consideramos esencial que los aprendices adquieran, durante sus clases de ELE, las herramientas lingüísticas básicas para poder identificar las marcas e indicadores propios del humor verbal. De esta forma, serán, posteriormente, capaces de interpretar las inferencias y resolver las incongruencias del texto humorístico. De lo contrario, es decir, si no están familiarizados con los usos y funciones del lenguaje humorístico, corremos el riesgo de que tomen el mensaje de forma literal y se frustren al no encontrarle el sentido. 


\section{Metodología y Diseño de la Investigación}

La metodología empleada para llevar a cabo esta investigación es de carácter hipotético-deductiva, lo que conlleva que sea, a su vez, exploratoria, descriptiva y contrastiva. En concreto, nos servimos de la transcripción de la muestra humorística, de un cuestionario $^{6}$ y de una plantilla de observación para extraer nuestros datos. El conjunto de métodos nos ha permitido analizar los recursos lingüísticos y extralingüísticos que los estudiantes son capaces de reconocer dependiendo de factores como su nivel de lengua o el grado de inmersión lingüística, entre otros.

\subsection{Herramientas estadísticas de análisis}

Primeramente, diseñamos una plantilla de observación con el propósito de analizar cómo se manifestaba el fenómeno de apreciación del humor en los escolares no nativos. Al utilizarla durante el primer visionado, los datos obtenidos corresponden a sus primeras reacciones ante los textos humorísticos - previas a que se vieran influidos por los comentarios y explicaciones del resto de los compañeros-. Atendimos a la expresión de sonrisas, risas, gestos faciales y comentarios del tipo "no lo entiendo", con el objetivo de asociar estas huellas a la aparición de los diversos indicadores y marcas durante el enunciado humorístico. Por otro lado, esta plantilla de observación nos sirvió también para estudiar cuál era la motivación y predisposición de los estudiantes hacia la actividad, lo cual influye, sin duda, a la hora de situarse en modo humorístico y apreciar el humor.

Un segundo instrumento para la obtención de datos fue la transcripción del texto audiovisual. Así, durante el segundo visionado, les dimos a cada estudiante una copia para que subrayaran en ella aquellos enunciados o palabras que reconocían como humorísticas. A raíz de los resultados obtenidos, elaboramos una hoja Excel en la que se indican los elementos lingüísticos y extralingüísticos más señalados.

Igualmente, una tercera herramienta para la recogida de datos fue un cuestionario online realizado a través de la plataforma digital Survio. Su carácter virtual posibilitó que las y los encuestados dispusieran de él en sus dispositivos electrónicos, así como en formato papel si no tenían acceso a la red. El cuestionario nos permitió recabar información sobre algunas de las variables que inciden en el desarrollo de las habilidades

\footnotetext{
${ }^{6}$ Cuando hablamos de "cuestionario" nos referimos a un formulario compuesto por una serie de ítems que no tienen por qué estar correlacionados y en el que no hay preguntas correctas o incorrectas.
} 
metapragmáticas del humor: la edad, el género, la procedencia, el nivel de español, la inmersión lingüística y el curso educativo. Asimismo, gracias a este cuestionario pudimos también estudiar aspectos relacionados con los procesos de comprensión y apreciación del humor, puesto que hubo varios ítems en los que los adolescentes tenían que escribir aquellas frases de la parodia que habían conseguido hacerles gracia y divertirles.

En cuanto al análisis de los datos, utilizamos Excel Office para recopilar la información en un libro de hojas de cálculo. A través de este software, hemos diseñado las distintas tablas y gráficas con las que se ilustrarán los resultados obtenidos. Del mismo modo, el programa estadístico R Project nos ayudó a efectuar un análisis ANOVA con los datos extraídos sobre la percepción del humor agresivo y la variable género.

\subsection{Rasgos principales de los participantes}

La adquisición y el desarrollo de la competencia humorística en hablantes no nativos conlleva, indudablemente, ser competente comunicativo y sociocultural. Por esta razón, decidimos tomar como objeto de estudio a jóvenes extranjeros que tienen en España su lugar de residencia y que están escolarizados en colegios internacionales. En concreto, la recogida de datos se llevó a cabo en las aulas de Bachillerato y Educación Secundaria Obligatoria (en adelante, ESO) de 8 centros educativos con programas plurilingües. Por consiguiente, los informantes del estudio son 71 adolescentes con edades comprendidas entre los 12 y los 18 años, que suman una media de 14'8 años.

La franja de edad que abarcan estos cursos escolares era la más ventajosa para el estudio, ya que, durante este período, los adolescentes -como ellos mismos reconocierontienen un contacto continuo con los géneros humorísticos a través de los chistes y memes en las redes sociales, las sitcoms y los vídeos de YouTube. Además, nuestra decisión de trabajar con este grupo de edad vino también motivada por las ideas presentadas en los trabajos de Orekoya et al. (65) y Zimmermann (6). De acuerdo con estos investigadores, es en la adolescencia cuando nuestro pensamiento alcanza mayor abstracción y se rige por principios lógicos. De tal modo que, de acuerdo con Martin, los participantes han adquirido ya la capacidad para detectar las incongruencias en el discurso y divertirse con chistes más complejos a nivel cognitivo y lingüístico (241). 
No obstante, de acuerdo con nuestra segunda hipótesis, la competencia humorística no solo se verá afectada por el factor edad, sino también por otras variables como la nacionalidad, el sexo, el nivel de lengua o el grado de inmersión lingüística. En este sentido, la procedencia de los estudiantes podrá favorecer, en algunos casos, el entendimiento y apreciación del humor verbal español. De hecho, para aquellas personas que hablan una lengua romance, como el francés, el portugués o el italiano, puede que les resulte más sencillo entender el contenido del mensaje. De igual modo, el uso habitual de géneros humorísticos como el Stand-Up Comedy o la parodia en países como Reino Unido y Estados Unidos podría favorecer la predisposición por parte de sus habitantes para situarse en modo humorístico.

Figura 1 País de procedencia de los participantes

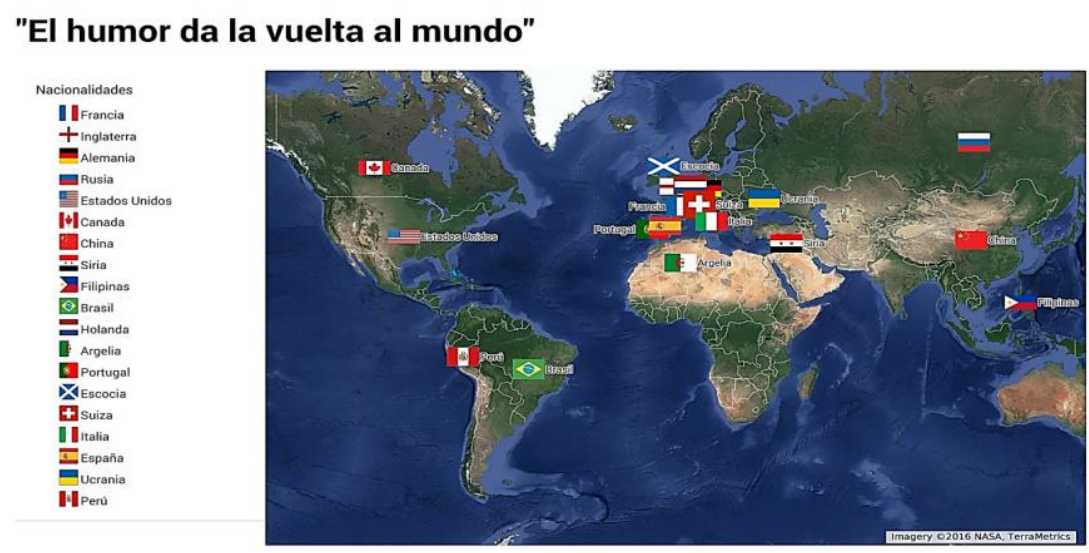

Como se observa en la figura 1 , los participantes procedían de todos los rincones del mundo. El grupo más numeroso lo conforman un total de 32 estudiantes procedentes de países anglófonos, seguidos por 14 rusófonos, 10 francófonos, 6 sinohablantes, 3 lusófonos, 2 germanohablantes, 2 neerlandófonos y otros 2 italianófolos. Asimismo, cabe señalar que se han descartado dos de las respuestas del estudio, ya que uno de los estudiantes procedía de un país de habla hispana y el otro había nacido en España, pese a tener raíces asiáticas.

Otro factor que se ha tenido en consideración en esta investigación es el género del alumnado. Pese a haber más similitudes que diferencias entre ambos sexos en cuanto la competencia y actuación del humor, como afirma Crawford (1413), conviene analizar en qué medida difiere la apreciación humorística de nuestras alumnas y alumnos atendiendo a esta variable. En esta línea, Robin Lakoff es una de las precursoras en introducir la variable 
del género en los estudios sobre el humor, al afirmar en su obra Language and Woman's Place que las mujeres carecían de sentido del humor (Lakoff 56). Sin embargo, esta afirmación, carente de comprobación empírica en su trabajo, fue desmontada poco tiempo después, y hoy en día sabemos gracias a rigurosos trabajos de investigación -como Jenkins (1985), Hay (2000), Kotthoff (2006) y Alvarado (2016)- que ambos sexos tienen sentido del humor, si bien es cierto que lo emplean de diversas formas y con funciones diferentes.

Del mismo modo, el grado de inmersión lingüística en el que se encuentran nuestros estudiantes será un elemento condicionante. De acuerdo con el Diccionario de Términos Clave $^{7}$ del Centro Virtual Cervantes, "se habla de inmersión total cuando la L2 es la lengua vehicular dominante en el currículo, y de inmersión parcial, cuando la primera y segunda lengua funcionan de manera alternativa como medio de comunicación en el aula". No cabe duda de que una situación en inmersión lingüística plena favorecerá el proceso madurativo de las habilidades metapragmáticas y la comprensión del humor en L2. En este sentido, Pastor Cesteros (2004) afirma que el uso de la lengua meta como vehículo de enseñanza influye en la adquisición de la competencia sociolingüística, en el desarrollo de la competencia comunicativa y, por tanto, en el aprendizaje de la L2.

\section{Gráfico 1 Número de asignaturas en español que cursan los estudiantes}

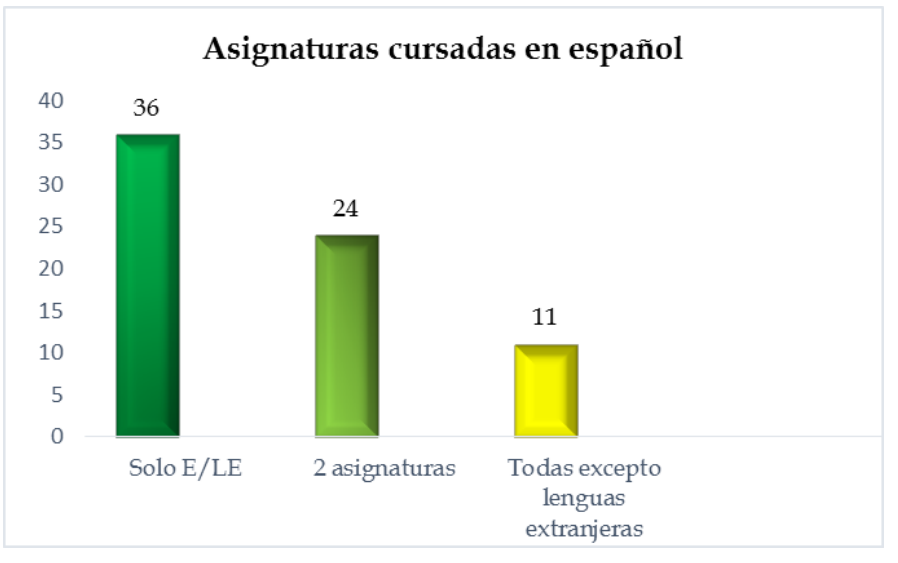

Así pues, observando el gráfico 1, podemos concluir que la gran mayoría de los encuestados no se encuentran en plena inmersión linguiística, ya que tan solo 11 de ellos cursan el grueso de sus asignaturas en español. No obstante, debemos reconocer que la

\footnotetext{
${ }^{7}$ Enlace de acceso a la definición: http://cvc.cervantes.es/ensenanza/biblioteca_ele/diccio_ele/diccionario/inmersionlinguistica.htm
} 
oportunidad de estar en contacto directo con la lengua fuera del aula favorece en gran medida el proceso el aprendizaje. De ahí que el nivel de los estudiantes se vea influenciado tanto por el grado de inmersión como por su relación con el entorno social ${ }^{8}$ fuera del aula.

En cuanto al nivel de lengua, los datos recogidos cifran en una mayoría simple a los estudiantes con nivel umbral B1 (35'2\%), seguidos por el alumnado con nivel B2 (28'2\%) y aquellos con nivel elemental A2 $\left(25^{\prime} 4 \%\right)$. Los porcentajes descienden en los niveles superiores, con tan solo un $10 \%$ de estudiantes con nivel avanzado $\mathrm{C} 1$ y un 1 '2\% con nivel maestría $\mathrm{C}^{9}$.

\subsection{Aplicación de la muestra audiovisual}

En primer lugar, en cuanto a la selección de la muestra, el ejemplo fue elegido con precaución para evitar incluir cualquier material cuyo contenido pudiera herir la sensibilidad de los alumnos o alterar el orden de la clase. De igual modo, durante la criba del material, tuvimos en cuenta factores que pudieran dificultar en exceso la comprensión. Entre los elementos que nos ayudaron a descartar determinadas muestras se encuentran: la dicción de los humoristas y su velocidad del habla, los juegos de palabras empleados y las referencias a elementos culturales desconocidos para los alumnos internacionales.

En nuestro propósito por facilitar la interpretación del contenido humorístico, seleccionamos una muestra de carácter audiovisual ${ }^{10}$; de manera que los estudiantes tuvieron la oportunidad de escuchar y ver cada una de ellas hasta un máximo de tres veces. El visionado inicial tuvo un objetivo de carácter bidireccional. Por un lado, quisimos que los alumnos y las alumnas tuvieran un primer contacto con el género humorístico y que alcanzaran una comprensión global. Por otro lado, fue durante esta reproducción cuando la investigadora anotó los diferentes indicios de apreciación del humor que revelaban los estudiantes con sus expresiones faciales, risas y comentarios. Seguidamente, con la

\footnotetext{
${ }^{8}$ Aunque la lengua meta está en el entorno inmediato del aprendiz y está expuesto a ella continuamente, varios de los docentes con los que pude compartir impresiones, manifestaron su inquietud por la situación de aislamiento a modo de burbuja en la que muchos de los estudiantes vivían dentro de sus comunidades.

${ }^{9}$ El único caso de nivel maestría C2 se dio en una adolescente italiana que llevaba viviendo en España de cinco a seis años y cursaba $2^{\circ}$ de Bachillerato en un centro educativo con inmersión lingüística plena.

${ }^{10}$ Creímos conveniente presentar un ejemplo de humor audiovisual con el fin de que los aprendientes se sirvieran de las marcas paralingüísticas y kinésicas empleadas por el humorista a la hora de captar el sentido y la intención del mensaje. Martin Peris (1991) subraya el carácter activo que conlleva el proceso de comprensión, ya que los oyentes deben interpretar el enunciado teniendo en cuenta determinados aspectos extralingüísticos propios del discurso oral.
} 
transcripción del material audiovisual delante, los escolares volvieron a ver el vídeo por segunda vez. En esta ocasión, se trataba de realizar una escucha más atenta para examinar la primera fase del humor, es decir, su reconocimiento. Por tanto, durante el segundo visionado, se les pidió que identificaran ejemplos de humor en el texto escrito. Finalmente, en caso de precisar de una tercera reproducción para llegar al entendimiento y apreciación de contenido, los informantes podían volver a ver el vídeo desde sus dispositivos electrónicos a la vez que realizaban el cuestionario.

En particular, el ejemplo de parodia fue extraída de un fragmento de Crackòvia, un programa de sátira deportiva de TV3. Nuestra elección vino marcada por el tema universal que representa el fútbol, ya que jugadores españoles como Sergio Ramos son mundialmente conocidos.

\section{Resultados}

Los resultados que analizaremos a continuación contestan a los interrogantes e hipótesis establecidas al comienzo de este trabajo. A la luz de los datos obtenidos, reflexionaremos sobre de qué modo han podido influir variables como el nivel de lengua, el grado de inmersión lingüística, la etnia, el sexo, o la edad.

\subsection{Resultados cuantitativos}

Desde una perspectiva cuantitativa, examinaremos los procesos de reconocimiento y comprensión del humor. Para ello, analizamos el número de ocasiones en las que nuestros informantes infirieron la comicidad de la parodia, a través de las marcas e indicadores, en las diferentes muestras humorísticas. Mediante diversas gráficas y tablas, ilustraremos los datos estadísticos extraídos de sus respuestas en la transcripción y el cuestionario.

\subsubsection{Identificación del blanco de burla}

La parodia es una manifestación humorística que contiene cierta similitud con la sátira, dado que se observa con claridad hacia quién va dirigida la burla, es decir, quién es el satirizado (Simpson, 2003: 123; Ruiz Gurillo, 2013: 257). Esta característica equivaldría al acto crítico propuesto Rossen-Knill y Henry (1997:736), acto que supieron reconocer la mayoría de nuestros estudiantes. De hecho, el $89 \%$ de los encuestados señalaron a Sergio Ramos como blanco de burla, y no es casual que el 11\% restante que erró en su respuesta 
presentara un nivel elemental A2 de español. Es evidente que, para reconocer la intención humorística del texto, se han de emplear determinadas habilidades metapragmáticas: inferir quién es el blanco de la burla requiere de una reflexión consciente tanto de qué se dice como de cómo se dice.

\section{Gráfico 2 Identificación del blanco de burla en la parodia}

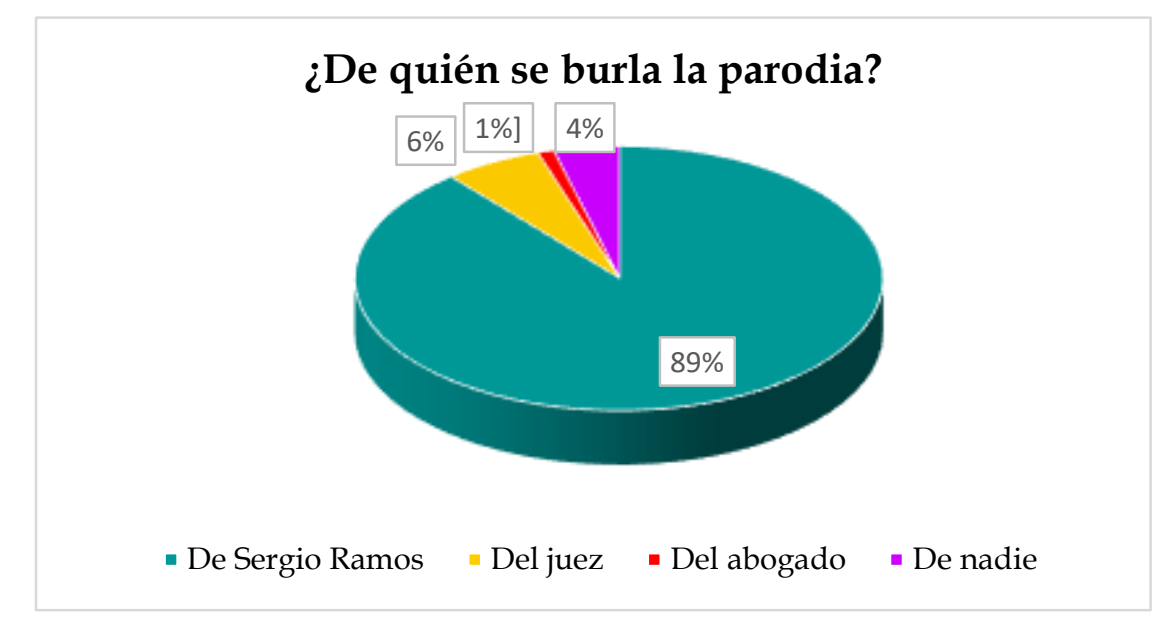

Siguiendo con los rasgos de la parodia verbal propuestos por Rossen Knill y Henry (1997), al acto crítico le acompañan la representación verbal intencional del objeto de la parodia y el alarde de la representación verbal. De modo que la coexistencia en la parodia de estos tres elementos origina un efecto humorístico o acto cómico. Entiéndase por representación verbal el contenido lingüístico a través del que se explicita la parodia y se exhibe el blanco de burla. Por su parte, el alarde de la representación verbal hace referencia al texto original que queda aquí parodiado (Ruiz Gurillo, 2012:87).

Figura 2 Elementos que componen la parodia (Rossen Knill y Henry, 1997)

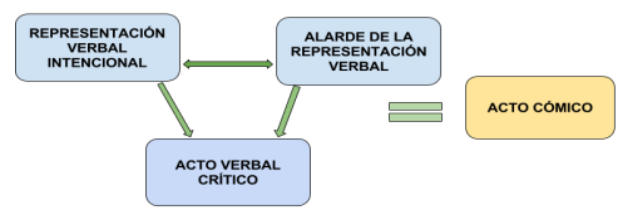




\subsubsection{La función del lenguaje en el reconocimiento y comprensión del humor}

Con el fin de lograr la comicidad que se persigue con este discurso humorístico, se emplean ciertas marcas e indicadores que actúan como elementos evaluativos, ya que además de colaborar en el efecto humorístico, transmiten también la actitud u opinión del emisor (Ruiz Gurillo, 2013: 262). De igual forma, estos elementos ejercen como huella metalingüística en el reconocimiento de la transgresión de los principios pragmáticos y en la resolución de la incongruencia. En este sentido, el alumnado que participó en nuestro estudio percibió gran parte de las pistas metalingüísticas integradas en la siguiente parodia:

\section{$(3)^{11}$}

$\mathrm{J}$ : en el acta consta que le dijo al árbitro/ eres un sin vergüenza llevas todo el día jodiéndonos/ ¿Tiene alguna defensa posible?

SR: que vaa/¡si no tengo defensa! mira/ Pepe lesionao/ yo estoy sancionao/ ((y aún peor la delantera))/ porque Cristiano no jugó $\rightarrow$

A: Sergio estate calladito que ya hablo yo ¿de acuerdo? estate calladito/ hablo yo/ yo sólo/ tú no dices nada ¿de acuerdo? (2')

A: ¿sí Sergio? ¿Estamos? (2’’)

A: ¿Sergio sí?

SR: $(($ achoo $)) \rightarrow$ ¡has dicho que no hablase!

(RISAS ENLATADAS)

A: mi cliente cree que la sanción de 5 partidos es exagerada/abrumadora $\uparrow$ por eso y con la venial su señoría $\S$

SR: $\S$ ¿la venia? ¿Quién es la venia?

\section{(RISAS ENLATADAS)}

A: es una forma de dirigirse al juez

SR: aah $\rightarrow$ la venia/ mucho gusto/ ¡la venia!

(RISAS ENLATADAS)

J: por favor diríjase a mí con un su Señoría

SR: su señoría la venia/ ¡qué nombre más original!

(RISAS ENLATADAS)

J: No no/ me llamo Francisco

SR: ah pues yo/ le llamaré Paco/ y su mujer le llamará PACO-MÉ

(RISAS ENLATADAS)

$\mathrm{J}$ : lo siento $\downarrow$ pero no puedo quitar la sanción de 5 partidos

A: no tenemos más remedio que acatar la sanción

SR: un segundo $\uparrow$ pido la palabra/¿no es suficiente castigo estar a 18 puntos del Barça?

(RISAS ENLATADAS)

SR: ¿acaso no es suficiente castigo aguantar la cara de vinagre que tiene Mou?

(RISAS ENLATADAS)

11 Texto transcrito según las claves del sistema Val.Es.Co 
SR: le voy a decir una cosa/ si nos pinchan/ ¿acaso no sangramos?

(RISAS ENLATADAS)

SR: si nos hacen cosquillas/ ¿acaso no nos reímos? y le diré más $\uparrow$ si nos envenenan ¿no nos morimos?

(RISAS ENLATADAS)

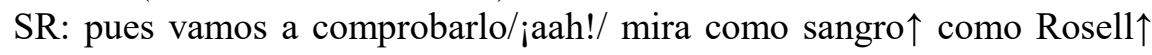
Sangro Rosell

Tras examinar las respuestas de nuestros informantes, la siguiente tabla y el gráfico ilustran los resultados que obtuvimos:

Tabla 1 Indicadores humorísticos identificados en la parodia por los estudiantes

\begin{tabular}{|c|c|c|c|}
\hline INDICADORES & EJEMPLO & $\begin{array}{l}\text { NÚMERO DE } \\
\text { RESPUESTAS EN } \\
\text { LA } \\
\text { TRANSCRIPCIÓN }\end{array}$ & $\begin{array}{l}\text { NÚMERO DE RESPUESTAS EN } \\
\text { EL CUESTIONARIO }\end{array}$ \\
\hline Fonema y alófono & $\begin{array}{l}\text { y su mujer le } \\
\text { llamará } \\
\text { PACO-MÉ }\end{array}$ & 33 & 22 \\
\hline Repetición & $\begin{array}{l}\text { ¿de acuerdo? } \\
\text { ¿sí Sergio? }\end{array}$ & 37 & 20 \\
\hline Paronimia & $\begin{array}{ll}\text { mira } & \text { como } \\
\text { sangro } \uparrow & \text { como } \\
\text { Rosell } \uparrow & \\
\text { Sangro Rosell }\end{array}$ & 18 & 9 \\
\hline Desautomatización & 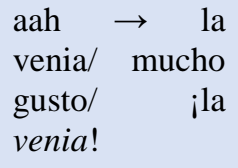 & 33 & 9 \\
\hline Polisemia & $\begin{array}{l}\text { J: ¿Tiene } \\
\text { alguna defensa } \\
\text { posible? } \\
\text { SR: que vaa/¡si } \\
\text { no tengo } \\
\text { defensa!/ }\end{array}$ & 27 & 8 \\
\hline Pseudoabarcador & $\begin{array}{l}\text { ¿no es } \\
\text { suficiente } \\
\text { castigo estar a } \\
18 \text { puntos del } \\
\text { Barça? ¿acaso } \\
\text { no } \\
\text { suficiente } \\
\text { castigo } \\
\text { aguantar la } \\
\text { cara de vinagre } \\
\text { que tiene } \\
\text { Mou? } \\
\end{array}$ & 18 & 3 \\
\hline
\end{tabular}




\section{Gráfico 3 Indicadores reconocidos por los estudiantes en la parodia}

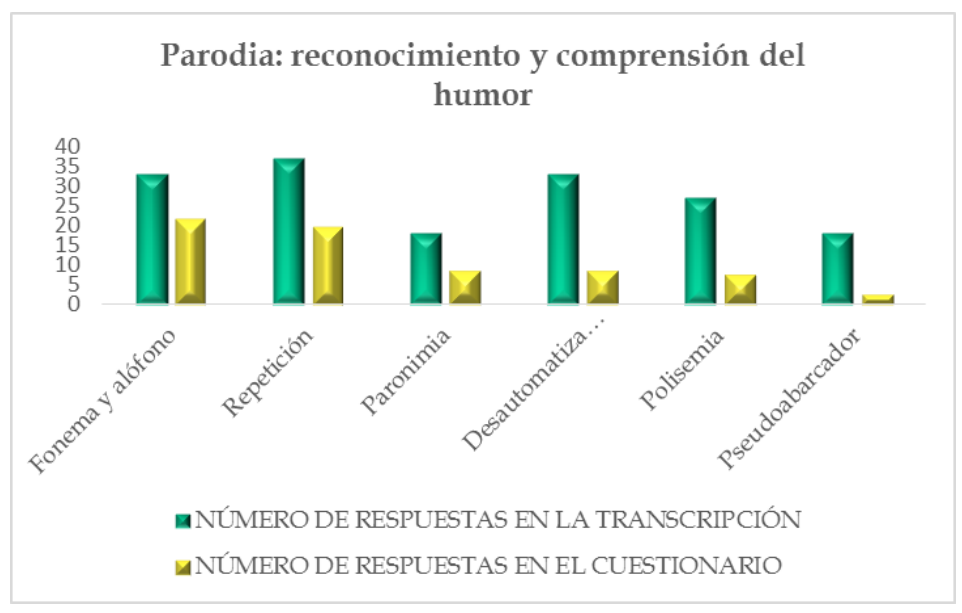

En primer lugar, la multiplicidad de referentes ante la palabra "defensa" fue señalada por 27 estudiantes en la transcripción, pero tan sólo escrita por 8 de ellos en la sección del cuestionario. Esta escasez de respuestas puede deberse a un déficit léxico que deriva en que no logren establecer las redes semánticas necesarias (Attardo \& Bell, 2010; Simarro Vázquez, 2013). Por lo que deducimos que la mayoría de los informantes conocían tan solo una o ninguna de las acepciones que recibe la palabra "defensa". En cuanto a las variables que hemos tenido en cuenta en el estudio, destaca la disparidad de género en las respuestas, ya que de esos 8 jóvenes que pasaron la barrera del reconocimiento y lograron entender el enunciado, 6 de ellos fueron varones.

Por el contrario, el fonema-alófono "Paco-me" fue de los indicadores con mayor porcentaje en reconocimiento y comprensión. Como se puede observar, este indicador recibió 33 identificaciones y 22 anotaciones posteriores, lo cual implica un gran dominio y conocimiento del registro coloquial, así como una alta competencia ortoépica. En este caso, el silabeo enfático que realiza el imitador fue la marca prosódica que facilitó que los informantes reconocieran este fenómeno fonológico.

Otro de los éxitos humorísticos de esta parodia fue la repetición de los marcadores de control del contacto “¿Sí?”, “¿de acuerdo?” y “¿estamos?”, junto con el silencio

\footnotetext{
${ }^{12}$ Se trata de un indicador humorístico que subvierte el principio de Informatividad al crear una ambigüedad entre lo que se cree que se pronuncia (fonema) y lo que realmente se pronuncia (alófono).
} 
prolongado por parte de Sergio Ramos, a razón de haberle pedido anteriormente que se mantuviera callado. En particular, dicho fragmento fue señalado por un total de 37 alumnas y alumnos en la transcripción y 20 de ellos lo escribieron además en el cuestionario. La repetición de marcadores propios del discurso oral actúa de gancho para desencadenar el remate "Achoo...has dicho que no hablase", y crean, de esta forma, una incongruencia humorística que se resuelve por medio del mecanismo lógico de razonamiento correcto basado en la coincidencia.

Por su parte, el fragmento en el que se produce la desautomatización del término jurídico "su Señoría la venia" fue únicamente registrado por nueve estudiantes, a pesar de haber sido un segmento identificado anteriormente por 33 escolares en el texto escrito. Por lo general, aquellos, que reconocieron la infracción del principio de manera que esta expresión marcada comete, tenían un nivel intermedio-alto de español y llevaban una media de 4 años en nuestro país. Asimismo, las marcas prosódicas fueron de ayuda para advertir la transgresión de la cualidad e interpretar el mensaje como humorístico.

Aunque, en nuestra opinión, los resultados más interesantes se registraron en la manifestación paronímica creada a partir del nombre propio "Sangro Rosell” para referirse al director del Fútbol Club Barcelona, Sandro Rosell. En este ejemplo, las marcas kinésicas tienen un papel principal en el desencadenamiento del efecto humorístico, ya que la paronimia se produce una vez que el imitador Sergio Ramos se autolesiona, grita y sangra. Así, la enunciación “¡aah! mira como sangro $\uparrow$ como Rosell $\uparrow$ Sangro Rosell” fue marcada por 18 estudiantes en la transcripción y empleada 9 veces como respuesta en el cuestionario. Los datos obtenidos con ambos instrumentos metodológicos manifiestan una significativa disparidad en el género. Como se observa en el siguiente gráfico, los varones manifestaron una mayor receptividad hacia este tipo de humor agresivo: 


\section{Gráfico 4 Reconocimiento y entendimiento del humor agresivo}

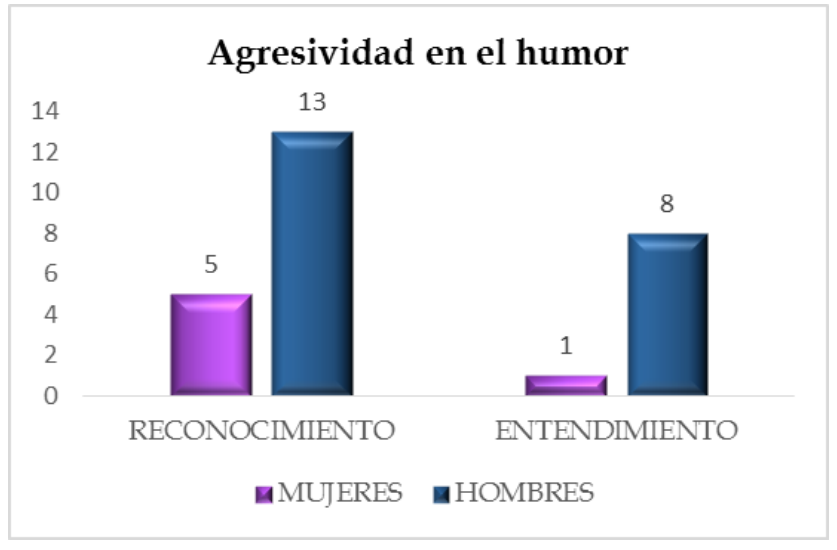

Así, observamos cómo el número de referencias a este enunciado, tanto en la fase de reconocimiento como en la de entendimiento, fueron realizadas por hombres en la mayoría de los casos. Quisimos entonces corroborar los estudios previos realizados por Kotthoff (2006), Timofeeva (2014) y Alvarado (2016) con respecto a la tendencia hacia el humor hostil y agresivo por parte del género masculino. Por ello, realizamos un análisis cuantitativo ANOVA, por medio del software estadístico R-Project, en relación con la variable género y los datos obtenidos en las transcripciones. Por un lado, nuestra hipótesis nula (H0) fue que no existían diferencias entre las mujeres y los hombres durante la fase de reconocimiento de humor agresivo, mientras que la hipótesis alternativa (H1) era que sí que había diferencias atendiendo a la variable género.

Figura 3 Resultados del análisis ANOVA realizado con R-Project

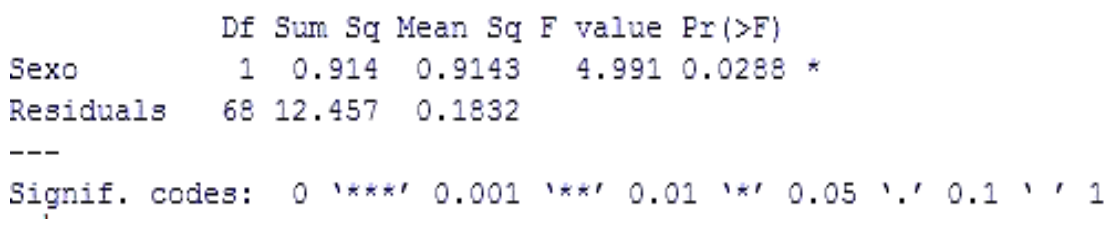

Este análisis demuestra que hay una mayor receptividad por parte de los varones hacia este tipo de humor. De hecho, obtuvimos un P-Valor de 0.0288 para un intervalo de confianza de 0.05. Estos resultados coinciden con los de las autoras mencionadas anteriormente y además confirman nuestra hipótesis acerca las diferencias en variables, como pueda ser género en relación con las fases del humor. No obstante, nuestra muestra no es lo suficientemente representativa para poder realizar afirmación de carácter genérico, 
sin duda, se necesitan investigaciones con una muestra de informantes más amplia que puedan ratificar nuestro estudio.

Por último, en el desenlace de esta parodia se hace una alusión literaria al monólogo de Shylock ${ }^{13}$ de la obra El Mercader de Venecia de William Shakespeare. Tanto Shylock como el imitador de Sergio Ramos formulan una serie de preguntas retóricas muy similares:

\section{Tabla 2 Comparación entre la obra literaria original y la parodia}

\begin{tabular}{|l|l|}
\hline Shylock en El Mercader de Venecia & Imitador de Sergio Ramos \\
\hline $\begin{array}{l}\text { ¿No comemos lo mismo? ¿No nos hieren } \\
\text { las mismas armas, no sufrimos las mismas } \\
\text { dolencias y nos curan los mismos }\end{array}$ & $\begin{array}{l}\text { ¿no es suficiente castigo estar a 18 puntos } \\
\text { del Barça? }\end{array}$ \\
$\begin{array}{l}\text { (RISAS ENLATADAS) } \\
\text { verano el mismo ¿No sufrimos en invierno y en }\end{array}$ & $\begin{array}{l}\text { SR: ¿acaso no es suficiente castigo } \\
\text { aguantar la cara de vinagre que tiene Mou? }\end{array}$ \\
$\begin{array}{l}\text { los cristianos? Y si nos pincháis, ¿No } \\
\text { sangramos? Si nos hacéis cosquillas, ¿No } \\
\text { reímos?, si nos envenenáis, ¿No } \\
\text { perecemos?" }\end{array}$ & $\begin{array}{l}\text { (RISAS ENLATADAS) le voy a decir una cosa/ si nos } \\
\text { pinchan/ ¿acaso no sangramos? }\end{array}$ \\
& $\begin{array}{l}\text { (RISAS ENLATADAS) } \\
\text { SR: si nos hacen cosquillas/ ¿acaso no nos } \\
\text { reímos? y le diré más } \uparrow \text { si nos envenenan } \\
\text { ¿no nos morimos? }\end{array}$ \\
\hline
\end{tabular}

Se trata, por tanto, de una metanarración en la que se refleja claramente el texto original parodiado, o en palabras de Rossen Knill y Henry (1997): el alarde de la representación verbal. Asimismo, la comicidad de este fragmento viene dada por el pseudoabarcador del término castigo que, sin embargo, no fue excesivamente reconocido como indicador humorístico. Tan solo 18 alumnos subrayaron la transcripción de esa escena y 3 de ellos la apuntaron cuando se les preguntó que fragmento les había hecho más gracia. Así pues, no es casual que todos los adolescentes que captaron este eco literario fueran anglosajones o estudiaran en colegios británicos ${ }^{14}$. Es evidente que la comprensión de este fragmento final requiere no sólo que sean competentes lingüísticamente, sino

\footnotetext{
13 Consúltese el monólogo interpretado por $\mathrm{Al}$ Pacino en el siguiente enlace: https://www.youtube.com/watch?v=_c8C7Dujn5E

${ }^{14}$ En uno de los centros, por ejemplo, los aprendices acababan de ver la versión cinematográfica de la obra $E l$ Mercader de Venecia.
} 
también que tengan unas determinadas nociones culturales.

\subsubsection{Factores condicionantes}

Para finalizar, cabe destacar dos factores que han condicionado en cierta medida los resultados obtenidos. De hecho, creemos conocer el porqué del contraste entre el volumen de respuestas en la transcripción y el cuestionario. Así pues, consideramos que las risas enlatadas de esta parodia fueron claves para la primera fase de reconocimiento. Se trata de una técnica muy típica en las sitcoms americanas y que, desde hace ya un par de décadas, también se emplea en el humor español. Como bien apuntan Kotthoff (2006: 7) y Bertero y Fung (2016: 383), este tipo de risas ayudan a predecir el humor y sirven al oyente no nativo como huella metapragmática para saber cuándo es apropiado reírse. En definitiva, las risas enlatadas actúan como marca extralingüística y contribuyen a contextualizar el fragmento dentro del marco humorístico. En esta línea, Nancy Bell comenta que "laughter that accompanies jokes would take away the burden of identifying the presence of a joke, leaving the student with only the task of determining the type" (254).

Por el contrario, consideramos que la disminución del volumen de respuestas durante la fase de entendimiento se debió a la variedad diatópica empleada. Pensamos que los estudiantes no están lo suficientemente familiarizados con la variedad del español peninsular meridional (andaluz), y su uso en esta parodia dificultó en cierta medida la comprensión del discurso. No obstante, consideramos que la parodia es una manifestación humorística apta para explotar didácticamente en el aula de E/L2, puesto que las risas y la fácil identificación del blanco de burla facilitan la comprensión de esta.

\subsection{Resultados cualitativos}

La metodología empleada en este estudio nos permite, además, realizar un análisis cualitativo de los resultados.

\subsubsection{La apreciación humorística de la parodia en el alumnado de E/L2}

Se confeccionó una plantilla de observación en la que decidimos no solo anotar sus señas de apreciación, sino que también tuvimos cuenta algunos otros elementos que podían influir en el fenómeno de la apreciación del humor. De este modo, registramos información sobre el tipo de centro educativo, el curso escolar, el nivel de español y el sexo del alumno. 
Prestamos también atención a factores internos ${ }^{15}$ del aprendizaje relacionados con la motivación y disposición del alumnado hacia la actividad. Aunque, sin lugar a duda, sus gestos, risas y comentarios fueron la clave para poder determinar qué mecanismos lingüísticos del humor lograban apreciar.

Una de las huellas de apreciación del humor que tuvimos en consideración fue la sonrisa, un factor que no había sido tenido en cuenta hasta hace muy poco. En los últimos años, la sonrisa se ha convertido en objeto de numerosos estudios como, por ejemplo, los trabajos realizados con la técnica de eye-tracking (Gironzetti, et.al., 2016). Sin embargo, se trata de una pista que no deja de llevar adherida cierta problemática, relacionada con la precisión y objetividad científica. Tener buen sentido del humor es un rasgo de la personalidad del ser humano que ha estado siempre altamente ponderado; por consiguiente, podemos en ocasiones tender a fingir apreciación, bien por salvaguardar nuestra imagen, o bien por estrechar lazos con el interlocutor si se trata de una conversación (Sacks, 1974; Hay, 2001; Bell, 2013). A pesar de esto, quisimos tomar la acción no verbal de sonreír como indicio de aprecio y respaldo al contenido humorístico expuesto.

De igual forma, decidimos tener en cuenta la risa como referente en la apreciación del humor, ya que es una de las respuestas más comunes ante algo que nos parece ocurrente o gracioso. De hecho, nadie duda de que la risa es el indicio contextual de apreciación del humor por excelencia y autores como Bergson (1911) ya hablaban de la risa como un fenómeno social que refleja un apreciamiento del humor. No obstante, este elemento también entraña cierta controversia, puesto que la risa no siempre significa que se comparte lo que dice el texto. A veces, la risa puede ser señal de sorpresa, vergüenza o distancia ante lo enunciado (Attardo,2003; Coates, 2007). Estas ideas quedan perfectamente resumidas en la siguiente cita de Helga Kotthoff:

When I tell someone a joke, I watch for signs of amusement. If the listener is willing to cooperate, s/he will let me finish, and then smile, laugh, or respond in some way to the humorous potential. If the listener did otherwise, she would define my joke as a dud. It is this dialogicity, which inhibits timid people, strangers, or people of lower status from joking in many contexts.

15 El Diccionario de términos clave de ELE (Martín Peris, 2008) señala como factores internos del aprendizaje: la motivación del aprendiente, sus necesidades y su estilo de aprendizaje. Mientras que determina como factores externos el contexto en el que el aprendizaje se produce, así como la enseñanza que intenta promoverlo. 
They fear the embarrassment of not eliciting the appropriate reaction (4).

En cuanto a las marcas de humor fallido, es oportuno fijar la atención en las expresiones faciales y comentarios, con el objetivo de advertir cualquier señal de incomprensión o rechazo ante lo dicho en el ejemplo. Aunque somos conscientes de que el silencio puede ser también una marca de humor fallido (Kotthoff, 2006; Bell, 2010), preferimos no tenerlo en cuenta porque, en esta ocasión, los participantes únicamente recibían input y no se les pidió que respondiesen ante lo dicho, es decir, la dialogicidad no era obligatoria.

\section{Gráfico 57 Registro del número de marcas de apreciación}

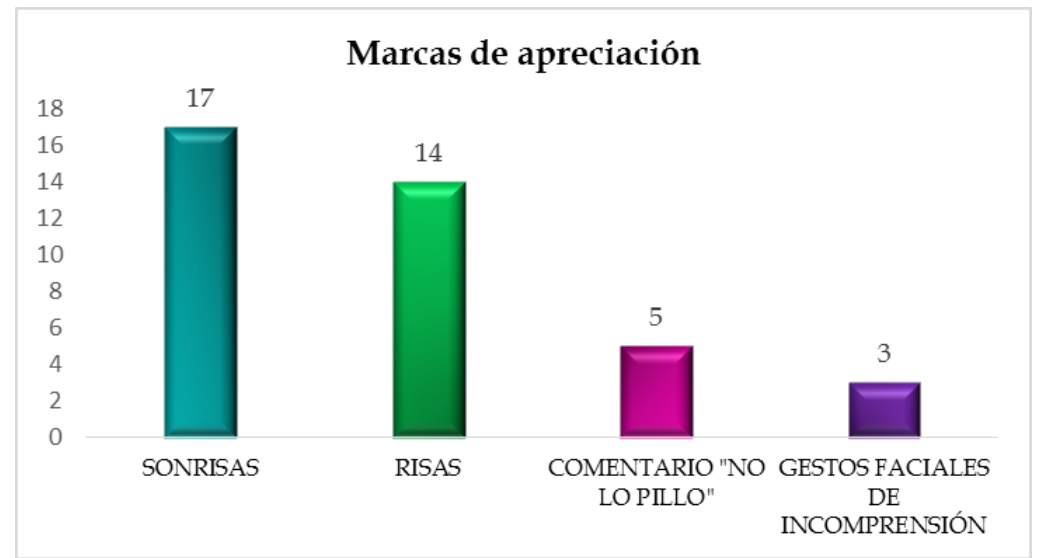

Por lo general, los estudiantes se mostraron receptivos ante la actividad, ya que esta implicaba salirse del libro de texto y de los contenidos previstos para el curso por un día. Durante el visionado pudimos percibir, con satisfacción, cómo disfrutaban con el vídeo y cómo, en su mayoría, respondían positivamente con sonrisas y risas. Asimismo, hubo ocasiones en las que los jóvenes manifestaron su incomprensión mediante sus expresiones faciales y comentarios del tipo “¿Qué? no lo entiendo” o "No lo he pillado”. Curiosamente, en este último caso, todos ellos fueron varones con un nivel umbral (B1) de español.

\section{Conclusión}

Este trabajo ha demostrado que, para lograr ser competente a nivel humorístico en una segunda lengua, se necesita ser también competente a nivel comunicativo, linguiístico y 
social. De hecho, hemos observado como la parodia, aun siendo una manifestación humorística planificada en la que las risas enlatadas y el blanco de burla facilitan el reconocimiento del humor, nuestros resultados muestran que la comprensión de este género es más complicada y está supeditada a factores contextuales, lingüísticos y sociales. De hecho, la burla suele ir dirigida a una personalidad, grupo social o tópico propio de la cultura del país; de modo que, si el oyente no nativo no identifica el blanco de burla, será imposible que se sitúe en un modo humorístico.

Ahora bien, creemos que la formación de la conciencia metalingüística y el desarrollo de la competencia sociopragmática se vería más rápidamente desarrollada si los docentes invitaran al alumnado a reflexionar sobre los diversos patrones lingüísticos que aparecen en muestras humorísticas como la parodia. Sin duda, el humor ha de ser un aliado en la labor docente, ya que un fragmento humorístico puede, en ocasiones, ser más significativo para el alumno/a que cualquier explicación teórica proporcionada en el manual de lengua. Sin embargo, si no empleamos con corrección esta herramienta, el humor puede resultar un distractor en el aula y arruinar toda nuestra sesión didáctica (Pomerantz \& Bell, 2011). Introducir material humorístico en las lecciones debe ser una acción meditada y estructurada que colabore en el desarrollo de la competencia comunicativa de nuestros estudiantes. Es necesario que, antes de implementar el humor en el aula, el profesorado conozca cuáles son los intereses y conocimientos culturales de sus estudiantes. Además, compartimos la idea de Deniere de que la selección del material humorístico debe también potenciar el desarrollo de la competencia intercultural, por lo que conviene alejarse de los estereotipos culturales con tal de evitar frustraciones y sensibilidades heridas en el aula (288).

En definitiva, estos resultados son la prueba fehaciente de que sí se debe trabajar el humor verbal en el aula de E/L2. Ahora bien, como hemos visto, el éxito de la muestra dependerá de factores tan diversos como la temática, el contenido sociocultural que incluya, la representación del humorista y por supuesto, la competencia lingüística del alumnado. 


\section{Bibliografía}

Alvarado Ortega, María. Belén. "Variability, adaptability and negotiability in conversational humor: A matter of gender". Metapragmatics of humor: Current Research Trends. Ed. L. Ruiz-Gurillo. Amsterdam: John Benjamins, 2016. 193214.

Attardo, Salvatore. y Raskin, Victor. Script Theory Revis(it)ed: Joke similarity and joke representation model. International Journal of Humor Research 4. 4-4 (1991): 437411.

Attardo, Salvatore. Linguistic theories of humor. Berlin: Mouton de Gruyter, 1994

Attardo, Salvatore. Humorous texts: A semantic and pragmatic analysis. Berlin: Mouton de Gruyter. 2001.

Attardo, Salvatore, Hempelmann, Christian F. y Di Maio, Sara. "Script oppositions and logical mechanisms: Modeling incongruities and their resolutions." Humor 15.1 (2003). 3-46.

Bell, Nancy. Learning about and through humor in the second language classroom. Language Teaching Research 13.3 (2009): 241-258.

Bell, Nancy y Attardo, Salvatore. Failed humor: Issues in non-native speakers' appreciation and understanding of humor. Intercultural Pragmatics 7.3 (2010): 423-447.

Bell, Nancy. "Humor scholarship and TESOL: Applying findings and establishing a research agenda". TESOL Quarterly 45.1 (2011): 134-159.

Bell, Nancy. Responses to incomprehensible humor. Journal of Pragmatics 57 (2013): 176189.

Bell, Nancy y Pomerantz, Anne. Humor in the Classroom: A Guide for Language Teachers and Educational Researchers. Routledge, 2015.

Bertero, Dario y Fung, Pascale. "A long short-term memory framework for predicting humor in dialogues." Proceedings of the 2016 Conference of the North American Chapter of the Association for Computational Linguistics: Human Language Technologies. 2016.

Crawford, Mary. Gender and humor in social context. Journal of Pragmatics 35.9 (2003): $1413-1430$.

Deneire, M. Humor and foreign language teaching. Humor: International Journal of Humor Research 8. 3 (1995): 285-298.

Gironzetti, Elisa, Attardo, Salvatore y Pickering, Lucy. "Smiling, gaze and humor in Conversation". Metapragmatics of humor: Current Research Trends. Ed. L. RuizGurillo. Amsterdam: John Benjamins, 2016.

Grupo GRIALE. ¿Estás de broma? 20 actividades para practicar la ironía en clase de ELE. Madrid, Edinumen, 2011.

Hay, Jeniffer. Functions of humor in the conversations of men and women. Journal of Pragmatics 32.6 (2000): 709-742.

Hay, Jeniffer. The pragmatics of humour support. Humor: International Journal of Humor Research 14. 1 (2001): 55-82.

Hess-Zimmermann. Is this a joke? Metalinguistic reflections on verbal jokes during the school years. E-JourALL, EuroAmerican Journal of Applied Linguistics and Language 3. 2 (2016) 3-21.

Jefferson, Gail. "On the organization of laughter in talk about troubles." Structures of 
Social Action: Studies in Conversation Analysis 346 (1984): 369.

Jenkins, Mercilee. "What's so funny? Joking among women." First Berkeley Women and Language Conference Berkeley, California. Proceedings. California: Women and Language Group Berkeley, 1985.

Kotthoff, Helga. "Gender and humor: The state of the art." Journal of Pragmatics 38.1 (2006): 4-25.

McGhee, Paul. E. Understanding and Promoting the Development of Children's Humor. Dubuque, Kendall Hunt Publishing, 2002.

Martín Peris, Ernesto. "La didáctica de la comprensión auditiva". Revista Cable 8 (1991): 1-11.

Martín Peris, Ernesto, Cortés, M. y Lopez, C. Diccionario de términos clave de ELE del CVC.Madrid:SGEL,2008. http://cvc.cervantes.es/ensenanza/biblioteca_ele/diccio_ele/indice.htm

Martin, Rod. A. The Psychology of Humor: An Integrative Approach. Burlington: Elsevier Academic Press, 2007.

Orekoya, O.S., Chan,. SS. y. Chik, M. P. "Humor and Reading Motivation in Children: Does the Tickling Work?” International Journal of Education 6.1 (2014): 61-72.

Pomerantz, Anne y Bell, Nancy. "Humor as safe house in the foreign language classroom." The Modern Language Journal 95 (2011): 148-161.

Ramos, Sergio. "Los mejores momentos de Sergio Ramos. Crackòvia. España: TV3, 2013. https://www.youtube.com/watch?v=pcFiohvIMXc\&t=113s

Rossen-Knill, Deborah F. y Henry, Richard. "The pragmatics of verbal parody." Journal of Pragmatics 27.6 (1997): 719-752.

Ruiz Gurillo, Leonor. "Para una aproximación neogriceana a la ironía en español”, Revista Española de Lingüística, 40. 2 (2010). 95-124.

Ruiz Gurillo, Leonor. La lingüística del humor en español. Madrid: Arco Libros, 2012.

Ruiz Gurillo, Leonor. \& Alvarado Ortega, María. Belén. (Eds.). Irony and Humor: from pragmatics to discourse. Amsterdam: John Benjamins, 2013.

Ruiz Gurillo, Leonor. "Parody as evaluation: about a television sketch in Spanish", Estudios de Lingüística Universidad de Alicante 27 (2013): 319-341.

Ruiz Gurillo, Leonor. "Infiriendo el humor. Un modelo de análisis para el español”, Revista Círculo de Lingüística Aplicada a la Comunicación 59 (2014): 148-162. http://pendientedemigracion.ucm.es/info/circulo/no59/rgurillo.pdf

Ruiz Gurillo, Leonor. "Metapragmatics of humor: Variability, negotiability and adaptability in humorous monologues". Metapragmatics of humor: Current Research Trends. Ed. L. Ruiz-Gurillo. Amsterdam: John Benjamins, 2016.

Sacks, Harvey. "An analysis of the course of a joke's telling in conversation". Explorations in the Ethnography of Speaking. Eds. R. Bauman y J. Sherzer. London: Cambridge University Press, 1974, 337-353

Schmitz, John Robert. "Humor as a pedagogical tool in foreign language and translation courses". Humor: International Journal of Humor Research, 15.1 (2002): 89-113.

Simarro Vázquez, María. "Los enunciados retroactivos humorísticos como medidores de la competencia léxico-semántica de hablantes de ELE". Humor, ironía y géneros textuales. Coords. M. B. Alvarado Ortega y L. Ruiz Gurillo. Alicante: Universidad, Servicio de Publicaciones, 2013. 83-116.

Simpson, Paul. On the Discourse of Satire: Toward a Stylistic Model of Satirical Humor. 
Amsterdam, The Netherlands: John Benjamins, 2003.

Suls, J. M. A. "two-stage model for the appreciation of jokes and cartoons: an information processing analysis". The Psychology of Humor, Eds. J.H. Goldsteins y P. E. McGhee. Nueva York: Academic Press, 1972. 81- 100.

Timofeeva, Larissa. El humor verbal en niños de educación primaria: presentación de un estudio. Femenismo/s, 24 (2014):195-219

Vivanco, Clotilde. "El acto humorístico en la enseñanza de las lenguas extranjeras". (Col.). Carlos Zenteno. Actas del XI Encuentro Nacional de Profesores de Lenguas Extranjeras en la Enseñanza Superior. Santiago, Chile: Universidad de Chile, 2000. 